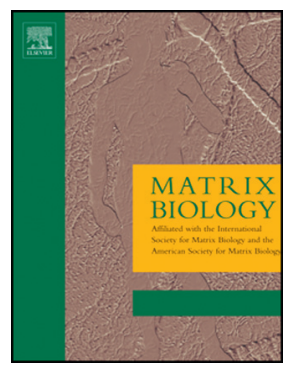

\title{
Drosophila type IV collagen mutation associates with immune system activation and intestinal dysfunction
}

\author{
Márton Kiss ${ }^{a}$, András A. Kiss ${ }^{a}$, Monika Radics ${ }^{a}$, Nikoletta Popovics ${ }^{a}$, \\ Edit Hermesz $^{\mathbf{b}}$, Katalin Csiszár ${ }^{\mathrm{c}}$ and Mátyás Mink ${ }^{\mathrm{a}}$ \\ a - Department of Genetics, University of Szeged, Középfasor 52, H-6726 Szeged, Hungary \\ b - Department of Biochemistry and Molecular Biology, University of Szeged, Középfasor 52, H-6726 Szeged, Hungary \\ c - John A. Burns School of Medicine, University of Hawaii, 1960 East West Road, Honolulu, HI 96822, USA
}

Correspondence to Mátyás Mink: mink@bio.u-szeged.hu.

http://dx.doi.org/10.1016/j.matbio.2015.09.002

Edited by R. Iozzo

\begin{abstract}
The basal lamina (BM) contains numerous components with a predominance of type IV collagens. Clinical manifestations associated with mutations of the human COL4A1 gene include perinatal cerebral hemorrhage and porencephaly, hereditary angiopathy, nephropathy, aneurysms and muscle cramps (HANAC), ocular dysgenesis, myopathy, Walker-Warburg syndrome and systemic tissue degeneration. In Drosophila, the phenotype associated with dominant temperature sensitive mutations of col4a1 include severe myopathy resulting from massive degradation of striated muscle fibers, and in the gut, degeneration of circular visceral muscle cells and epithelial cells following detachment from the BM. In order to determine the consequences of altered BM functions due to aberrant COL4A1 protein, we have carried out a series of tests using Drosophila DTS-L3 mutants from our allelic series of col4a1 mutations with confirmed degeneration of various cell types and lowest survival rate among the col4a1 mutant lines at restrictive temperature. Results demonstrated epithelial cell degeneration in the gut, shortened gut, enlarged midgut with multiple diverticulae, intestinal dysfunction and shortened life span. Midgut immunohistochemistry analyses confirmed altered expression and distribution of BM components integrin PSI and PSII alpha subunits, laminin gamma 1, and COL4A1 both in larvae and adults. Global gene expression analysis revealed activation of the effector AMP genes of the primary innate immune system including Metchnikowin, Diptericin, Diptericin B, and edin that preceded morphological changes. Attacin::GFP midgut expression pattern further supported these changes. An increase in ROS production and changes in gut bacterial flora were also noted and may have further enhanced an immune response. The phenotypic features of Drosophila col4a1 mutants confirmed an essential role for type IV collagen in maintaining epithelial integrity, gut morphology and intestinal function and suggest that aberrant structure and function of the COL4A1 protein may also be a significant factor in modulating immunity.
\end{abstract}

(c) 2015 The Authors. Published by Elsevier B.V. This is an open access article under the CC BY-NC-ND license (http://creativecommons.org/licenses/by-nc-nd/4.0/).

\section{Introduction}

The polarization and function of the epithelia including mucosal epithelia is determined and supported by a specialized form of the extracellular matrix $(\mathrm{ECM})$, the basal lamina or basement membrane (BM). In various tissues and body compartments, the BM also fulfills diverse functions including separation, absorption, sensation and secretion [1]. The BM is made of numerous components with a predominance of type IV collagens. Mammals, including humans, harbor three pairs of type IV collagen genes (COL4A1-6). The inherited disorder of renal, ocular and cochlear basement membranes associates with mutations of the X-linked COL4A5gene in the majority of patients with Alport syndrome, whereas mutations in the autosomal COL4A3 and COL4A4 genes are responsible for the symptoms of Alport syndrome in 
about $20 \%$ of patients [2,3]. In the mammalian BM, the dominant type IV collagen units are trimers with (COL4A1) ${ }_{2}$ COL4A2 composition.

Clinical manifestations associated with mutations of COL4A1 include perinatal cerebral hemorrhage and porencephaly [4], hereditary angiopathy, nephropathy, aneurysms, and muscle cramps (HANAC) [5], ocular dysgenesis, myopathy and Walker-Warburg syndrome [6]. The latest reports also demonstrated systemic tissue degeneration

A
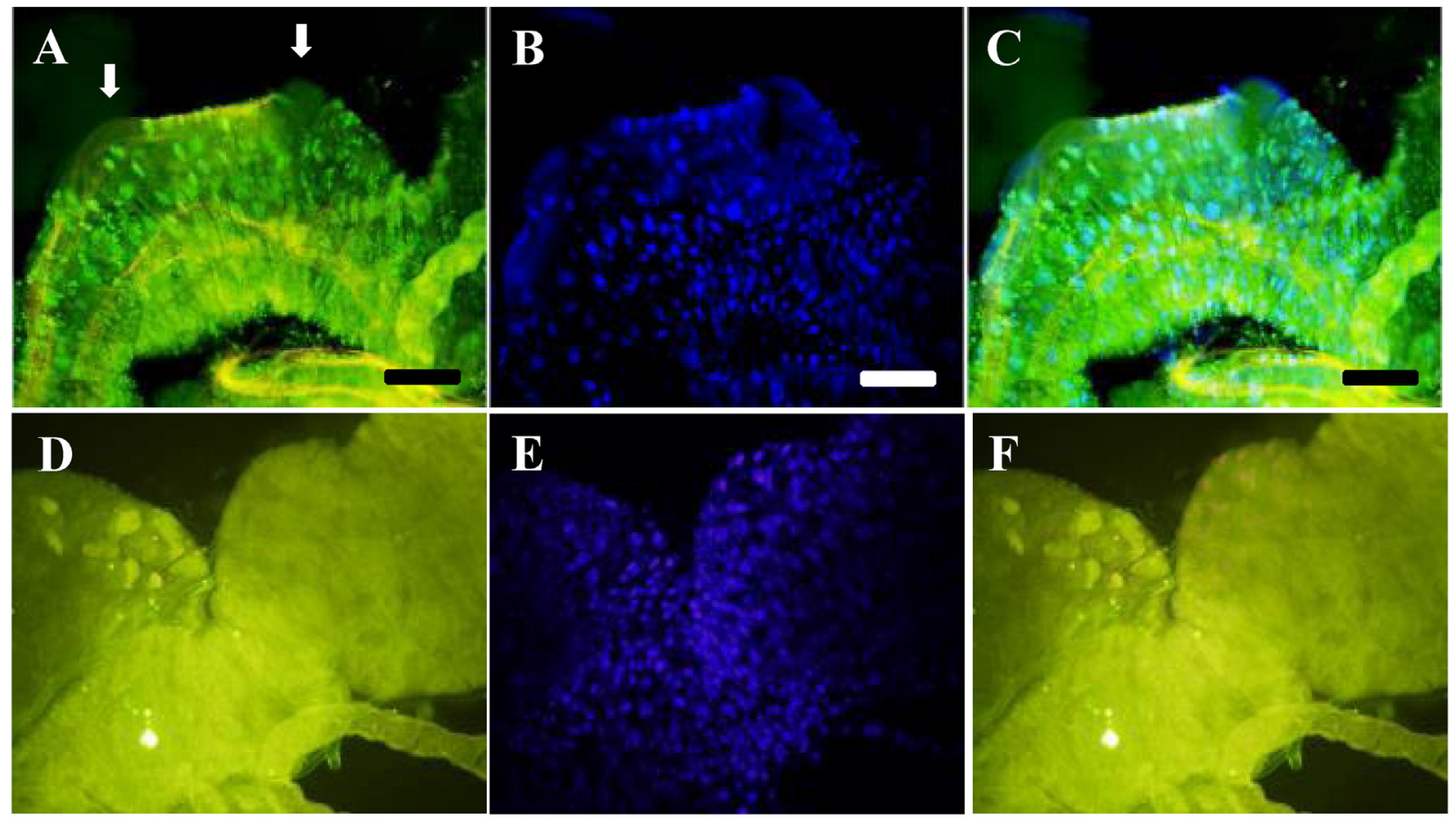

B
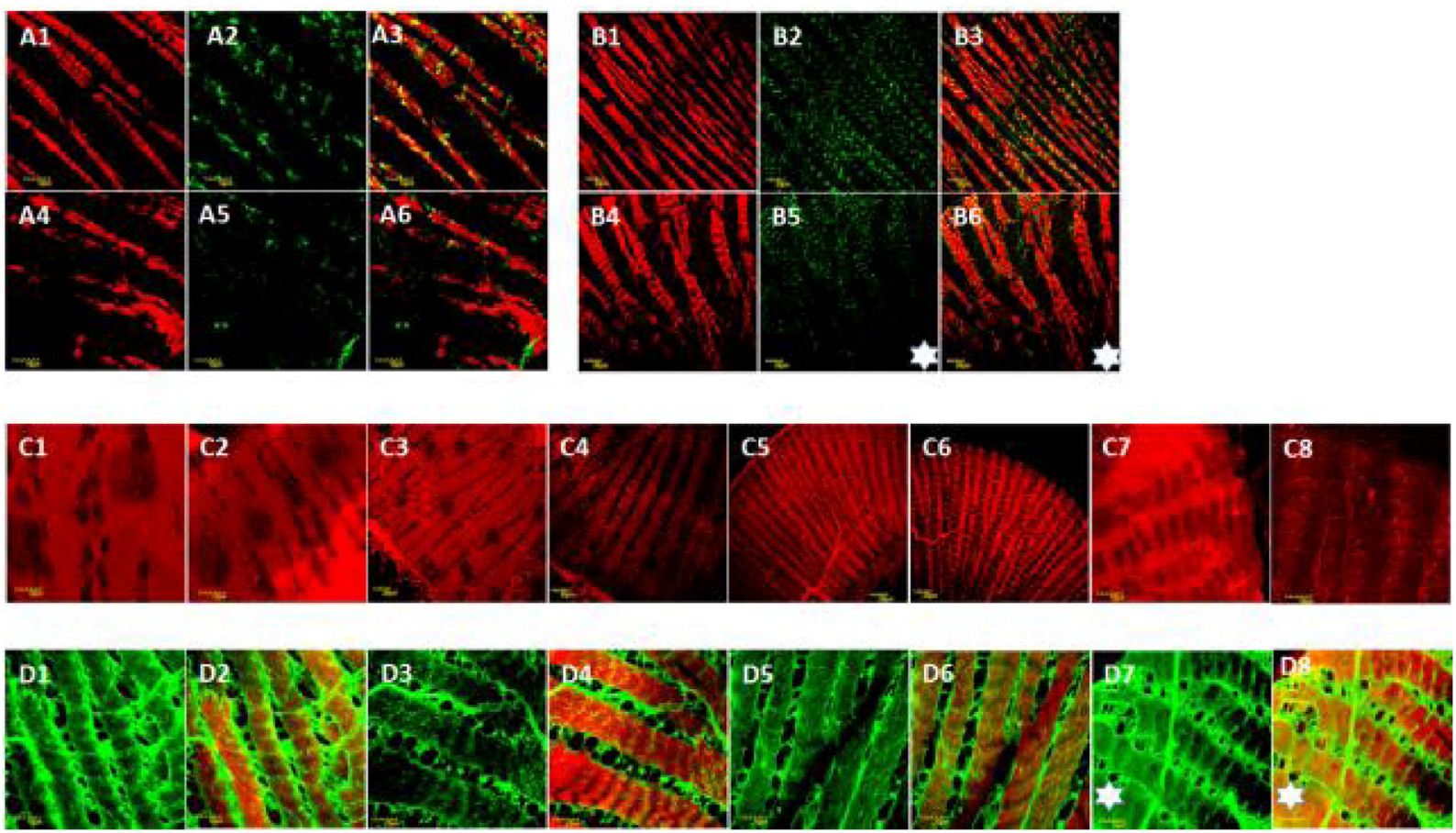

Please cite this article as: M. Kiss, et al., Drosophila type IV collagen mutation associates with immune system activation and intestinal dysfunction, Matrix Biol (2015), http://dx.doi.org/10.1016/j.matbio.2015.09.002 
and pleiotropy associated with COL4A1 mutations and confirmed that the phenotypes of COL4A2 mutations are phenocopies of COL4A1 gene mutations [7].

We have recently reported an allelic series of conditional, dominant, temperature-sensitive (DTS) mutations within the type IV collagen gene (col4a1) in Drosophila. The col4a1 mutant heterozygotes are viable and fertile at permissive temperature $\left(20^{\circ} \mathrm{C}\right)$, but perish at restrictive temperature $\left(29^{\circ} \mathrm{C}\right)$. The phenotype associated with mutations of col4a1 include severe myopathy resulting from massive degradation of striated muscle fibers [8], degeneration of the circular visceral muscle cells and the epithelial cells of the gut that occur following detachment from the BM [9]. While there are signs of repair, the capacity of the scavenger system, and/ or the kinetics of cell renewal and regeneration fail to keep up with the ongoing cell degeneration in these mutants $[8,9]$.

The mucosal epithelia of the gastrointestinal tract are constantly challenged by the microbiome resulting in various types of interactions including commensalism, symbiosis and pathogenicity [10]. Drosophila is one of few animal models in which host-microbe interactions in the gut have been studied [11]. The gut-associated bacterial community in Drosophila is scarce, harboring 5-10 bacterial phylotypes [12] and among these five dominant commensal species: Lactobacillus plantarum, Lactobacillus brevis, Acetobacter pomorum, Gluconobacter morbifer and Commensalibacter intestini [13,14]. Drosophila antimicrobial defense is facilitated by the phagocytosis of pathogenic microorganisms and by the synthesis of antimicrobial peptides (AMPs). Epithelial defense also relies on the generation of reactive oxygen species (ROS) [15-17].

Activation of the immune system by overexpression of AMPs, including Diptericin, Cecropin, Drosomycin, Defensin, Attacin and Metchnikowin, as demonstrated in caudal hypomorphic RNAi mutants, results in restructuring of the commensal bacterial population with the dominance of the pathogenic Gluconobacter morbifer accompanied by degradation of gut epithelial cells and high mortality of the host $[13,14]$ suggesting a role for deregulated expression of AMPs in epithelial cell degeneration and gut pathogenesis. Overexpression of AMPs was similarly linked in aging flies to dysfunction of the intestinal barrier that normally permits the absorption of nutrients and solutes and hampers host contact with harmful entities $[18,19]$. Compromised intestinal barrier function was also noted in big bang $(b b g)$ null Drosophila mutants that in the absence of the BBG protein that is localized in gut epithelial septate junctions, demonstrated reduced lifespan and chronic inflammation of the anterior midgut epithelium [20]. While some of the col4a1 mutation-associated phenotypic features were consistent with chronic inflammation, inflammatory and immune-activation mechanisms have not been explored either in Drosophila col4a1 or in association with mouse or human COL4A1 mutations [5,6,7,13,14].

In order to determine if mutated col4a1 and the resulting aberrant BM induced cell degeneration, compromised gut mucosal epithelial functions and activated the immune system, we have carried out a series of tests in mutant animals including evaluation of larval development, gut morphology, integrity of epithelial and visceral muscle cells of the gut, degree of cell death, immunohistochemistry of BM proteins in the midgut, global gene expression changes, expression values of the activated AMP effector genes of the primary innate immune system, changes in AMP protein Attacin::GFP levels in the midgut, and ROS production. We have also analyzed changes in the composition of the gut bacterial flora and the tolerance of bacterial load as indicators of modified immune functions, and recorded changes in life-span. From our allelic series of col4a1 mutations, we have selected for these analyses the DTS-L3 mutant based on the confirmed degeneration of various cell types and lowest survival rate among the col4a1 mutant lines at restrictive temperature of $29{ }^{\circ} \mathrm{C}[8,9]$.

\section{Results}

\section{Aberrant gut morphology, cell degeneration and altered expression of BM components in the midgut of col4a1 mutants}

Massive cell degeneration that we have described earlier $[8,9]$ was confirmed at restrictive temperature $\left(29^{\circ} \mathrm{C}\right)$ in the midgut of col4a1 adult mutant flies carrying the dominant temperature-sensitive DTS-L3 allele. Additionally, the following phenotype changes were noted: L3-stage DTS-L3 mutant larvae were shorter in length $(4.9+/-0.3 \mathrm{~mm})$ at $29^{\circ} \mathrm{C}$ compared to values $(5.5+/-0.4 \mathrm{~mm})(\mathrm{p}=0.032)$ at $20^{\circ} \mathrm{C}$ and to wild-type larvae at either temperatures. Gut alterations

Fig. 1. A. Fluorescence micrographs demonstrating TUNEL positivity in larval midgut. A: TUNEL-positive midgut of a DTS-L3 L3-larva incubated at $29^{\circ} \mathrm{C}$; arrows point to diverticulae and to protruding peritrophic matrix. B: DAPI, C. Merge. D, E, F: Midgut of a DTS-L3 L3-larva incubated at $20^{\circ} \mathrm{C}$ appears TUNEL-negative. Scale bars: $50 \mu \mathrm{m}$. B. Actin (red) and integrin (green) staining of the L3 larval midgut visceral muscle cells. A1: Actin, A2: Integrin, A3: Merge, wild-type; A4-6, mutant, same order, $20^{\circ} \mathrm{C}$. B1-3, wild type, B4-6 mutant, $29^{\circ} \mathrm{C}$. Asterisks refer to areas of lower integrin expression. C1, C2: Laminin expression (red) in the midgut of $\mathrm{L} 3$ control/mutant larvae, $20{ }^{\circ} \mathrm{C}, \mathrm{C} 3, \mathrm{C} 4$, at $29^{\circ} \mathrm{C}$. C5, C6: Laminin expression in the midgut of control/mutant adults, $20^{\circ} \mathrm{C}, \mathrm{C} 7, \mathrm{C} 8,29^{\circ} \mathrm{C}$. Note decreased laminin expression in $\mathrm{C} 4$ and $\mathrm{C} 8$. 
included shorter gut (1.5+/-0.4 arbitrary units) compared to those of wild-type larvae $(1.9+/-0.3$ arbitrary units) irrespective of the incubation temperature. In the transparent larvae, pathological gut morphology included enlarged midgut with diverticulae at several sites (Fig. 1A), whereas the midgut of the control larvae had a flat surface at $29^{\circ} \mathrm{C}$ (Fig. 1D).
Fluorescent light microscopic inspection revealed additional protruding sites/diverticulae along the midgut. The nuclei within areas of the diverticulae proved TUNEL-positive involving nearly all nuclei (Fig. $1 \mathrm{~A}-\mathrm{C}$ ). Areas of the diverticulae, furthermore, lacked cellular organization and presented a protruding peritrophic matrix (Fig. $1 \mathrm{~A}-\mathrm{C}$ ) while the midgut of the
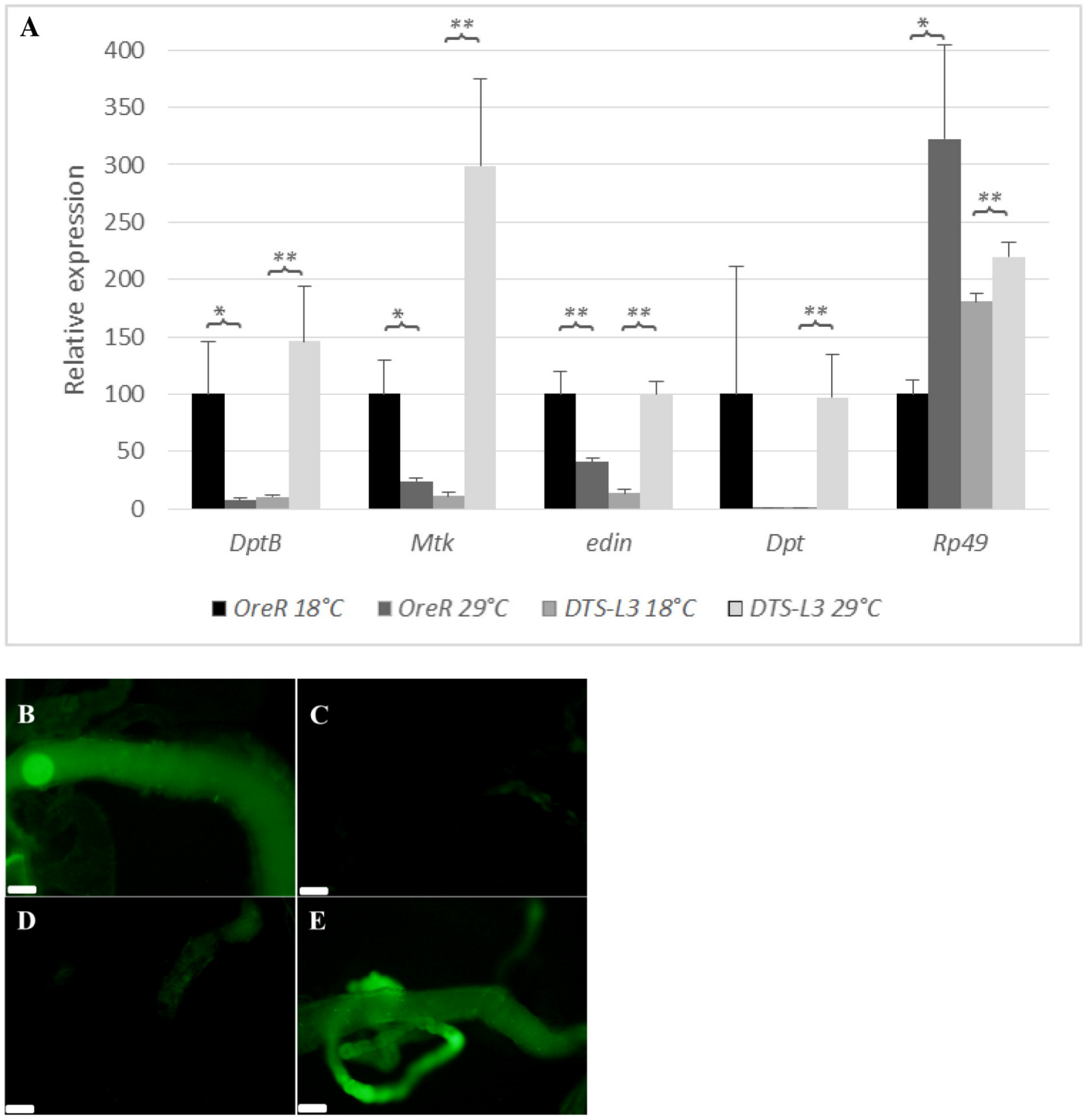

Fig. 2. A. QRT-PCR validation of the expressional data of selected AMP genes. Metchnikowin (Mtk), Diptericin (Dpt), Diptericin $B(D p t B)$ and edin in DTS-L3 mutants, following a three-day shift to restrictive temperature ${ }^{*} p<0.05 ;{ }^{* *} p<0.01$. Expression values of the ribosomal protein 49 gene (Rp49) served as an internal control. ATT::GFP expressions in control (Fig. 2B) and mutant (Fig. 2C) flies at permissive temperature and at $29^{\circ} \mathrm{C}$ (Fig. 2B, D, respectively). Scale bars: $100 \mu \mathrm{m}$. Note the opposite expressional levels in the wild-type and mutant animals. 
mutant larvae reared at $20{ }^{\circ} \mathrm{C}$ did not show these features (Fig. 1D-F).

The expression and distribution of BM components integrin PSI and PSII alpha subunits, laminin gamma 1 and collagen COL4A1 were analyzed in the midgut using immonohistochemistry (Fig. 1B). Panels A1-3 present the visceral muscle cells of the midgut in control L3 larvae, A4-6 correspond to mutant midgut stained for integrins (green) and actin (red) at $20^{\circ} \mathrm{C}$. In control larvae, punctate integrin staining localized to the Z-disks while in mutants slightly less integrin expression was detected at this location. This tendency was more apparent in larvae reared at $29^{\circ} \mathrm{C}$ where large areas in the midgut of the mutant lacked integrin expression (asterisks, Fig. 1 B1-3 vs. B4-6). The same integrin expression pattern was observed in the midgut of adult mutant animals (Supplementary Fig. S1). Laminin gamma 1 was similarly reduced in the midgut of mutant L3 larvae (Fig. 1B, C3 vs. C4, red) and in the midgut of adult animals (Fig. 1B, C7 vs. C8, red) at $29^{\circ} \mathrm{C}$ and appeared as a diffuse staining in mutants $(\mathrm{C} 4, \mathrm{C} 9)$. COL4A1 staining accumulated in the sarcoplasm in the midgut of the mutant at $20^{\circ} \mathrm{C}$ (Fig. 2B, D3, D4, green) and indicated a diffuse, irregular staining pattern at $29^{\circ} \mathrm{C}$ (Fig. 2B, D7, D8, green, asterisks). These results corroborated our earlier data and confirmed a systemic, multi-organ phenotype in col4a1 mutants at restrictive temperature $[8,9]$.

\section{Overexpression of AMP genes of the primary innate immune system and elevated oxidant production in col4a1 DTS-L3 mutants}

DNA-array experiments were carried out to detect col4a1 mutation-associated gene expressional alterations in DTS-L3 mutant animals compared to wild-type control flies at both permissive and restrictive temperatures [8]. Most of the upregulated genes in the mutants at restrictive temperature were related to immunological functions including host defense response, antibacterial humoral response, and innate immune response (Supplementary Table 1). The expression of certain peritrophic matrix constituents (CG10154 and CG10725), as well as genes for digestive tract enzymes (CG17234 and CG31091) and a transmembrane transport protein (CG9825) were also elevated in the mutants. In wild type Oregon files, the expression patterns for all of these genes followed an opposite tendency and were downregulated at elevated temperature. We have also found a gene, CG13043 that was significantly downregulated in the mutant but was upregulated in wild type flies. The CG13043 gene encodes a Retinin domain protein (pfam04527), considered to be a cuticle protein with yet unknown function that is expressed in the trachea and the testis.

Genes His3 and Hsp22 were upregulated and one of the peritropic matrix protein genes, obst- $B$, was downregulated in both mutant and control samples likely as part of an orchestrated response to elevated temperature. BobA and Dup99B were also found underexpressed in both mutants and controls. BobA is responsible for Notch signaling, while Dup99B plays a role in oviposition. Both genes seemed to be temperature-regulated regardless of mutational status.

In col4a1 mutants, AMP genes of the primary innate immune system Metchnikowin, Diptericin, Diptericin B, Attacin A, Attacin C and edin (elevated during infection) showed upregulation in the mutants whereas downregulation in controls at restrictive condition at statistically significant $(p<0.01)$ levels. Results of the DNA-array experiments are summarized in Supplementary Table 1 and gene expression data were deposited in the GEO-Database under accession number GSE52579.

DNA-array expression values for the AMP genes were validated by QRT-PCR. The data corroborated values of the DNA-array results and confirmed low levels of expression at $20^{\circ} \mathrm{C}$ and significantly increased expression of Metchnikowin (Mtk), Diptericin (Dpt), Diptericin B (DptB) and edin in mutants following a three-day shift to restrictive temperature, while in control animals, these genes showed significant downregulation at $29^{\circ} \mathrm{C}$ (Fig. 2A).

Expression changes at protein level were established utilizing the Drosophila transgenic line carrying the Attacin::Green Fluorescent Protein (GFP) fusion under the control of the Att promoter [39]. Att mRNA expression was downregulated $\log 2=-2,29$-fold in wild-type control animals, whereas upregulated in mutants log $2=3,19$-fold following a shift to $29^{\circ} \mathrm{C}$ (Supplementary Table 1). Protein levels followed the pattern of transcriptional alterations. In controls, reduced amount of ATT::GFP (Fig. 2B, D), whereas in mutant animals increased amount of fusion protein was detected at $29^{\circ} \mathrm{C}$ (Fig. 2C, E).

In order to evaluate whether an increase in oxidant production also occurred in mutants, we have measured the concentrations of two oxidants peroxynitrite and hydrogen peroxide at both $20^{\circ} \mathrm{C}$ and $29^{\circ} \mathrm{C}$. In mutants, peroxynitrite production was detected at higher concentrations compared to controls regardless of the temperature (Fig. 3). Hydrogen peroxide concentrations increased statistically significantly $(p<0.05)$ in the mutant and were slightly elevated in control animals at $29^{\circ} \mathrm{C}$ (Fig. 3).

\section{Bacterial species and counts are reduced in the col4a1 mutant gut niche}

In order to determine if altered expression of the primary/innate immune system had functional consequences in mutant animals, we have analyzed the gut microbial flora and compared to normal controls. Amplification and sequencing of the $16 \mathrm{~S}$ ribosomal RNA genes of the commensal bacteria from both 


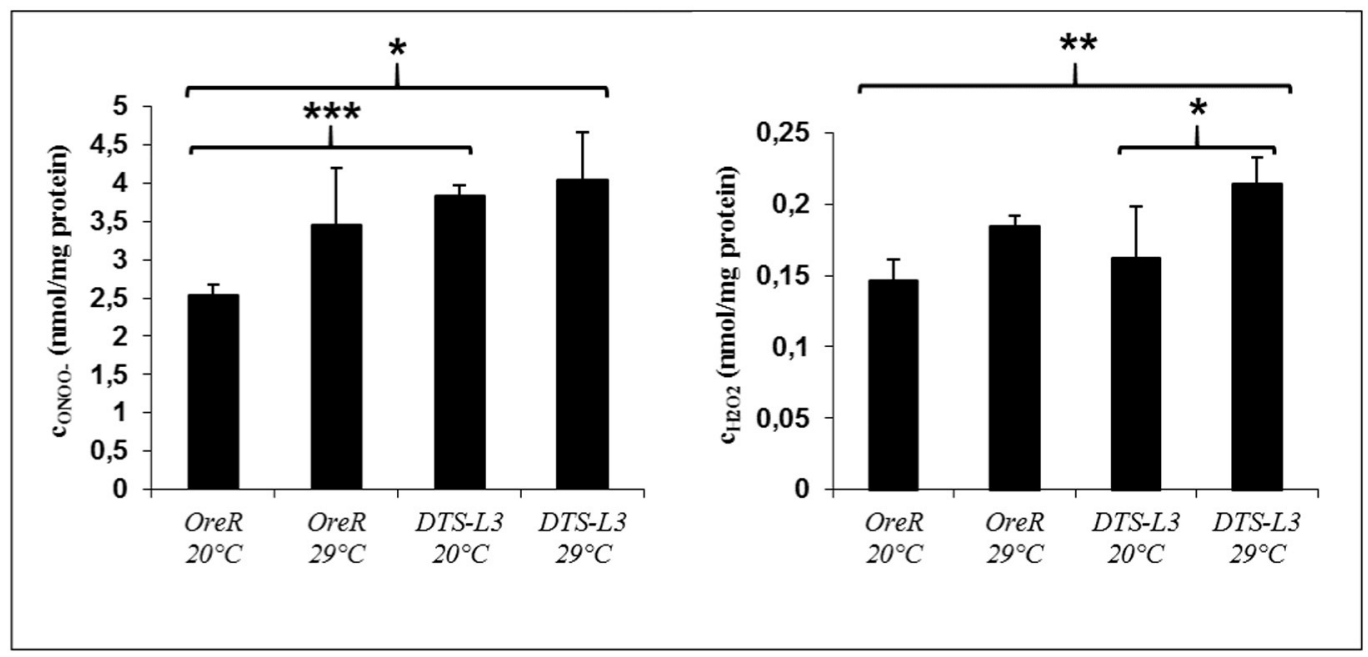

Fig. 3. Measurements of $\mathrm{ONOO}^{-}$and $\mathrm{H}_{2} \mathrm{O}_{2}$ concentrations in $\mathrm{nmol} / \mathrm{mg}$ protein units in control and mutant animals at both permissive and restrictive temperatures. All values are presented as means \pm S.D. ${ }^{*} p<0.05,{ }^{* *} p<0.005,{ }^{* *} p<0.001$.

mutants and controls kept at elevated temperature for 3 days was carried out. BLAST searches of the nucleotide sequences of the amplified fragments identified two common bacterial phylotypes, Acetobacter cerevisiae and Lactobacillus plantarum in both lines. As the composition of the intestinal microbiome changes dynamically and is dependent on environmental factors, we also cultivated both mutant and wild-type flies on starch-based medium. Under these conditions, beyond Acetobacter and Lactobacillus a third cultivable member of the gut-associated microbial community appeared in form of red colonies that, upon morphologic evaluation and ITS sequence analysis, proved to be yeast Rhodothorula species. Following a shift to sucrose-based food, Rhodothorula disappeared from the gut niche. The titers of the microbial community were also determined in the gut of mutant and wild-type flies. The number of colony-forming units
(CFU) was $\sim 10^{5}$ per gut both in mutants and controls at $20^{\circ} \mathrm{C}$. At $29^{\circ} \mathrm{C}$, statistically significant reduction ( $p=0.015)$ in CFU values was recorded in wild-type flies and while not statistically significant, a decrease of CFU was also noted in mutants. The elevated oxidant concentration (Fig. 3) and increased rate of oxidative reactions at $29{ }^{\circ} \mathrm{C}$ were consistent with the observed decrease in CFU values of the intestinal microbiome (Fig. 4).

Importantly, in mutants, the Acetobacter/Lactobacillus ratios revealed a statistically significant shift toward Acetobacter, a feature that was already present at permissive temperature (Fig. 4).

\section{Increasing the bacterial dose is lethal at $29^{\circ} \mathrm{C}$}

The lifespan of the wild-type control and mutant flies is 105 days that does not differ significantly at permissive temperature. Rearing both lines at $29^{\circ} \mathrm{C}$

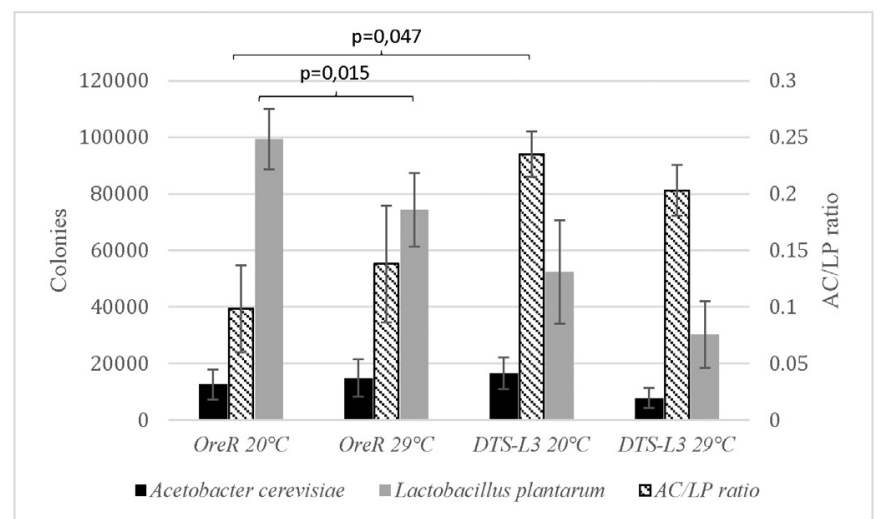

Fig. 4. CFU values of Acetobacter cerevisiae and Lactobacillus plantarum in DTS-L3 and wild-type flies incubated at $20^{\circ} \mathrm{C}$ and $29^{\circ} \mathrm{C}$. Left y axis: colony count/gut; right y axis: A. cerevisiae/L. plantarum (AC/LP) ratios. 


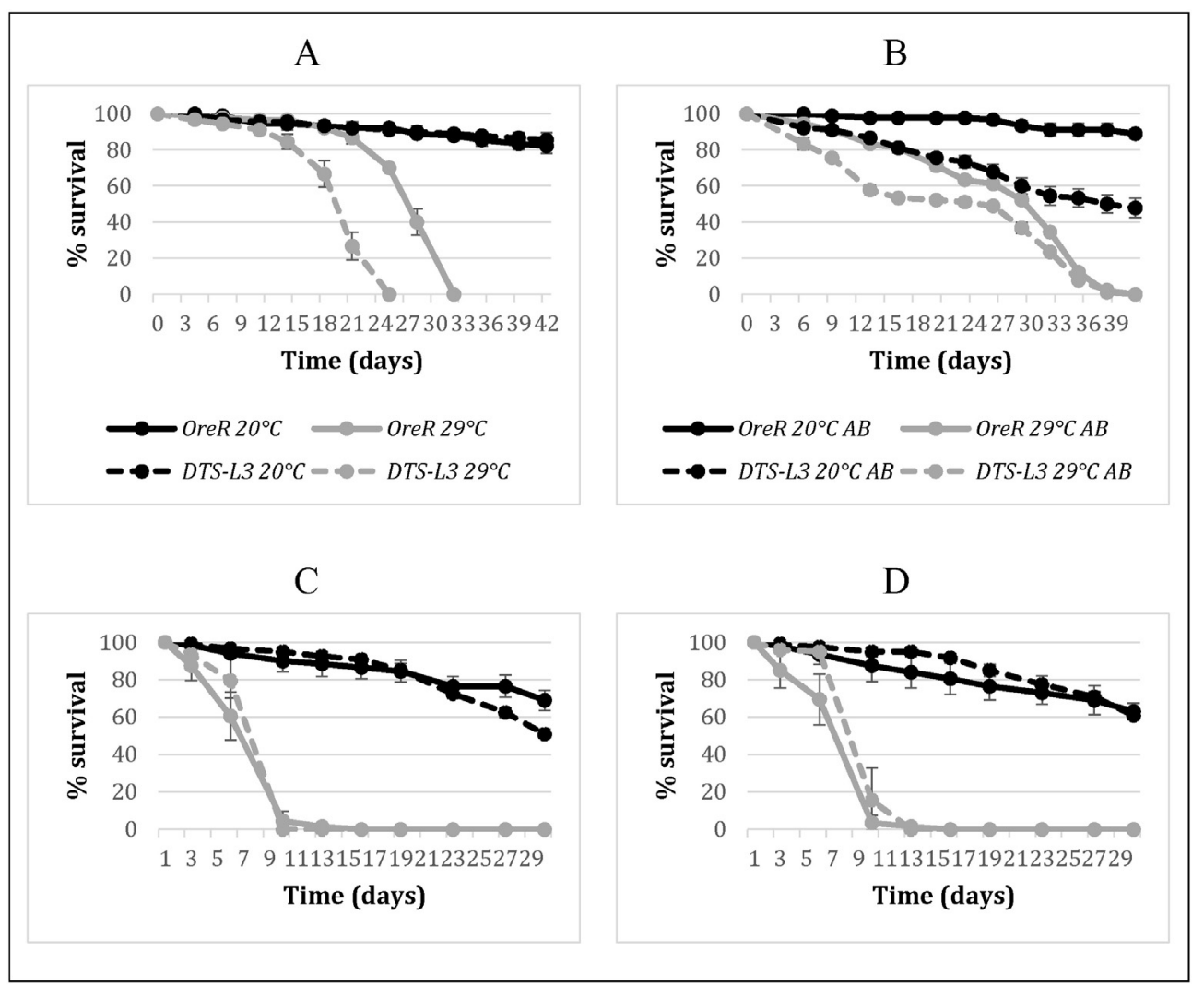

Fig. 5. A: Survival of mutant and control flies, expressed as percentage of escapers, at both permissive and restrictive temperatures on normal food. B: Effect of antibiotic treatment on survival, C: Survival on Acetobacter cerevisiae, D: Survival on Lactobacillus plantarum. Legends to Figures $\mathrm{C}$ and D are the same as in Figure A.

reduced longevity of the wild-type flies to 32 days, whereas to 25 days of mutants (Fig. 5A).

To test the hypothesis that increased levels of the normal gut-associated bacteria may affect the lifespan, isolated microbiotes were orally fed to both mutant and wild-type flies. The survival rates of the orally infected flies revealed that neither Lactobacillus plantarum nor Acetobacter cerevisiae influenced the life span of either mutant or wild-type flies at $20^{\circ} \mathrm{C}$ (Fig. 4C, D), and the CFU values in the gut remained in the range observed in the uninfected population $\sim 10^{5} \mathrm{CFU} /$ gut (not shown). In contrast, at $29{ }^{\circ} \mathrm{C}$, both mutant and wild-type flies succumbed following ten days of oral infection (Fig. 4C, D) irrespective the bacteria used with dramatically increased intestinal CFU values, exceeding 1$4 \times 10^{6} \mathrm{CFU} /$ gut (not shown). These results showed that a young population of both mutant and wild-type flies maintain relatively constant intestinal CFU values at $29{ }^{\circ} \mathrm{C}$ and suggested that the composition and titer of the intestinal microbial community does not contribute remarkably to the pathology of the young DTS-L3 mutants, and that their reduced lifespan (25 versus 32 days) may be due to other factors.
In aging flies, in contrast, increased bacterial loads have been demonstrated by several laboratories and were suggested to contribute to the death of the animals [19,21-23]. Premature aging and death of the col4a1 mutant flies might be caused by earlier onset of increased bacterial load, therefore we anticipated extended life span of the mutants by rearing them on antibiotics at restrictive temperature. Indeed, lifelong clearing of bacteria increased the life expectancy of both mutant and control lines up to 38 days at $29^{\circ} \mathrm{C}$ (Fig. 5B). These results were consistent with previous experimental evidence [19,21-23] and confirmed that elevated bacterial load is malign in aging flies but did not explain why col4a 1 mutant flies still succumbed significantly earlier on antibiotics-free medium.

\section{Premature loss of intestinal integrity in col4a1 mutants}

Detachment and TUNEL-positive staining of the gut epithelial and visceral muscle cells in the DTS-L3 mutants at restrictive condition (Fig. 1), [9] indicated loss of intestinal integrity. Therefore, we tested barrier function of the alimentary tract by monitoring 

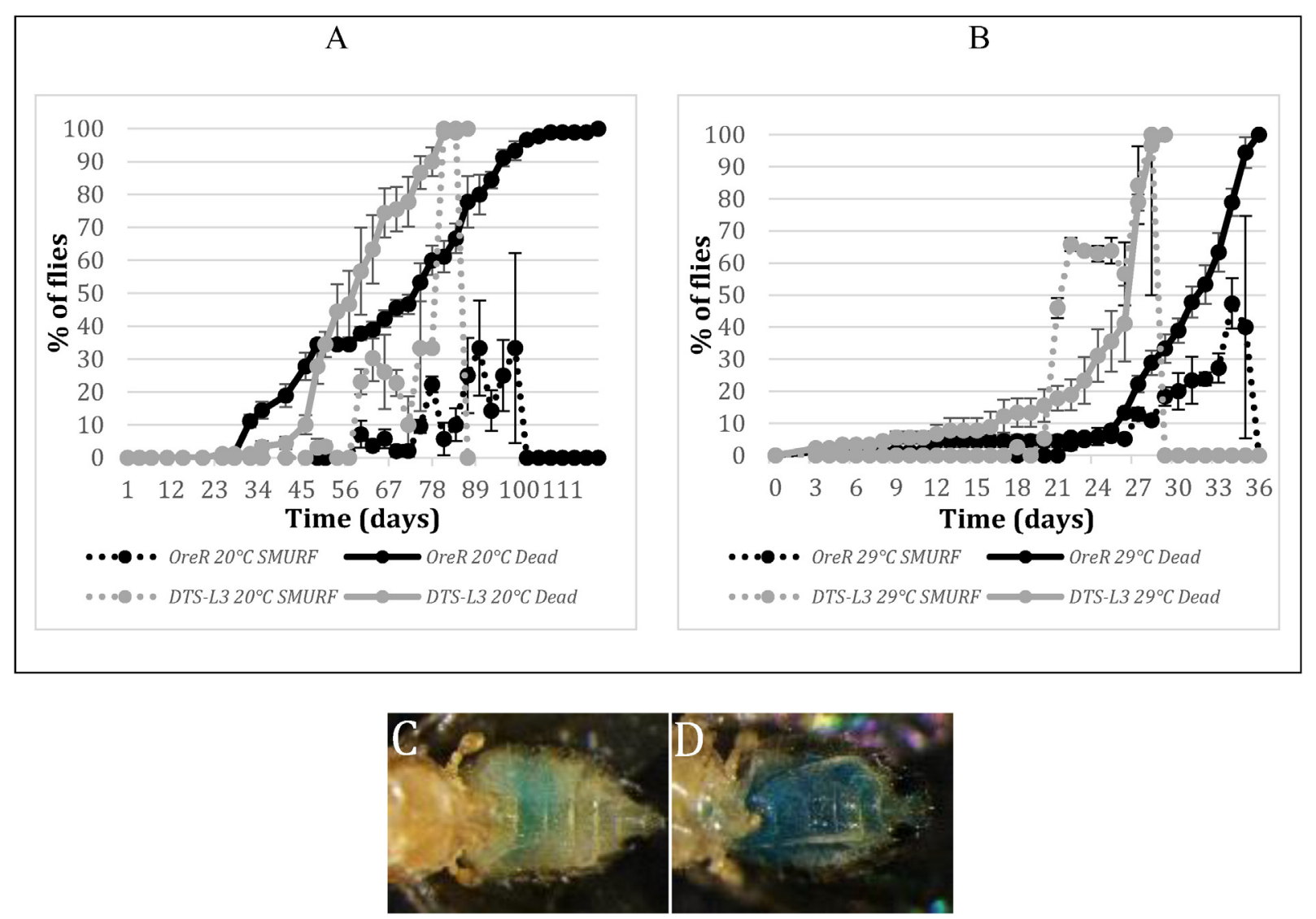

Fig. 6. A: Onset of SMURF phenotype and survival of DTS-L3 and wild-type flies incubated at $20{ }^{\circ} \mathrm{C}$, and $\mathrm{B}$ : at $29{ }^{\circ} \mathrm{C}$. C: Staining of the intact intestine in young mutant. D: SMURF phenotype marked by staining of the hemolymph in an aging mutant fly with abrupt onset of degradation of intestinal integrity and death of the animals at 20-22nd day of incubation.

the onset of the SMURF phenotype, i.e. penetrance of non-absorbable blue food dye into the hemolymph in both mutants and controls. At $20^{\circ} \mathrm{C}$, intestinal dysfunction did not occur until 57 days of incubation in either mutants or controls with no significant differences between the two lines (Fig. 6A). Incubation at $29^{\circ} \mathrm{C}$, however, resulted in an abrupt onset of the SMURF phenotype in DTS-L3 mutants (Fig. 6B) between 20 and 22 days followed by immediate death of the animals (Fig. 6A). Intestinal dysfunction in wild-type flies did not occur within this time-frame (Fig. 5C, D). These results suggested that premature aging of the DTS-L3 mutants was due to dysfunction of the intestinal barrier that also explained their short lifespan.

\section{Discussion}

The components and three-dimensional structure of the ECM provide a microenvironment that regulates basic cellular functions. Aberrant expression and structure of the ECM during tissue remodeling or pathological processes activate the immune system and influence cell survival. Degraded fragments of the
BM also modulate the activities of both resident cells and infiltrating immune cells [24]. Collagen type IV fragments resulting from BM degradation by metalloproteinases were implicated in the synthesis of antimicrobial peptides (AMPs) and stimulation of innate immune responses during insect metamorphosis [25]. Endogenously derived danger/alarm signals, including collagen type IV fragments, also stimulated immune cells in lepidoptera [26]. While modulated genetic elements for components of the BM (laminin, nidogen/entactin, perlecan, integrins and crosslinking lysyl oxidases) are available in Drosophila [27-29], the specific role of aberrant BM and mutated collagen type IV in modulating immune functions has not been explored in this model.

We have demonstrated earlier that in temperaturesensitive col4a1 Drosophila mutants altered BM structure and function cause degradation of skeletal and visceral muscle cells resulting in severe myopathy $[8,9]$. Results of the current study confirmed that mutated col4a 1 also causes degeneration of epithelial cells of the gut at restrictive temperature. Following continuous incubation of mutants at $29^{\circ} \mathrm{C}$ from egg deposition, morphological changes manifested in 
shorter larvae and the development of severe gut pathology including shorter gut, enlarged midgut, and multiple diverticulae collectively leading to intestinal dysfunction and shortened life span.

In adult animals, three days of temperature challenge and the resulting massive cell degradation as part of an early response, was sufficient to activate the immune system manifested in the overexpression of AMP genes Metchnikowin, Diptericin, Diptericin B, and edin. In contrast to mutants, wild-type control flies downregulated AMP synthesis at $29^{\circ} \mathrm{C}$ in agreement with the reported downregulation of Metchnikowin in flies kept at $29^{\circ} \mathrm{C}$ [30].

Multiple lines of evidence suggest a direct relationship between AMP overexpression, apoptosis of gut epithelial cells, and mortality reported for both caudal Drosophila mutants and aging animals $[13,18,19]$. In col4a1 mutants, temperature-induced initiation of epithelial cell detachment from the BM, activation of the immune system, and subsequent intestinal dysfunction appeared to be the primary causes of their abrupt and premature death. Elevated oxidant concentrations may have additionally promoted epithelial damage and intestinal dysfunction.

A shift in gut bacterial species and decrease of the CFU values were temperature-dependent responses irrespective of col4a1 mutation. Immune deficiency pathway-mutants have a contrasting higher gut CFU values, indicating that the basal level of antimicrobial peptides effectively regulate bacterial density [31]. Reduced bacterial count observed in controls with reduced AMPs and in DTS-L3 mutants with overexpressed AMPs suggested that factors other than AMPs contributed to the maintenance of gut homeostasis at elevated temperature.

Already present at permissive temperature, in mutants the Acetobacter/Lactobacillus ratios revealed a statistically significant shift toward Acetobacter. The acetic acid produced by Acetobacter effectively modulates insulin/insulin-like growth factor signaling in Drosophila that regulates developmental rate, body size, energy metabolism, and intestinal stem cell activity [32]. Thus the predominance of Acetobacter in the gut flora of col4a1 mutants may modify additional regulatory mechanisms that contribute to the phenotype.

In DTS-L3 col4a1 mutants, visceral muscle cell degeneration [9] has at least two severe consequences. First, during regeneration of the gut epithelium by the intestinal stem cells, visceral muscle cells that surround the epithelium produce epidermal growth factor Vein [31] a process that may be severely compromised in mutants. Second, compromised visceral muscle cells may cause motility-associated disorders, including life-threatening chronic intestinal pseudo-obstruction (CIPO) [33]. Therefore, Vein deficiency and CIPO may also be contributing factors to premature aging and death of mutants.
Considering the present data a question arises: Why human patients and mice with COL4A1 mutations do not develop chronic inflammation and prominent activation of the immune system? The mammalian genetic alteration differs from the col4a1 Drosophila mutation in several aspects. The homologous Drosophila mutations do not affect viability, fertility or cause detectable BM-associated cell degeneration at permissive temperature. Strong systemic phenotype occurs only following temperature stress. Stress-induced phenotype was similarly observed in $\mathrm{Col}_{4} \mathrm{a}^{-1-}$ mouse embryos. Col4a1 or Col4a2 null homozygotes were lethal at a developmental phase when the embryonic heart started to beat and put the blood vessels under pressure [34].

Our results with a Drosophila col4a1 conditional dominant mutant line support the suitability of this model to study mechanistic elements of the human COL4-associated condition, mutant phenotypic features confirm an essential role for type IV collagen in maintaining epithelial integrity, gut morphology, and intestinal function, and suggest that aberrant structure and function of the COL4A1 protein may be a significant factor in modulating immunity.

\section{Experimental procedures}

\section{Drosophila maintenance, RNA isolation, labeling, and DNA-array experiments}

Wild-type Oregon flies and col4a1 mutant stock with the DTS-L3 allele were maintained at $20^{\circ} \mathrm{C}$ or $29^{\circ} \mathrm{C}$ on yeast-cornmeal- sucrose-agar medium supplemented with propionic acid and hydroxymethylbenzoate to avoid fungal infections. In the starch-based food, sucrose was replaced by wheat flour. To test the effect of antibiotics we used a combination of ampicillin and tetracycline, dissolved in $50 \%$ ethanol, mixed into the medium to a final concentration of $500 \mu \mathrm{g} / \mathrm{ml}$ and $50 \mu \mathrm{g} / \mathrm{ml}$, respectively. The vials were prepared a day before use and were changed in every 6 days. Intestinal barrier dysfunction was monitored by mixing 30 -fold diluted stock solution of the blue food dye Patent Blue V/E131 in the liquid medium and recording the onset of the diffusion of the dye from the gut into the surrounding tissues (SMURF phenotype).

The col4a1 mutant stock was kept heterozygous over the CyRoi balancer chromosome. Prior to RNA isolation the dominant temperature-sensitive DTS-L3 mutant and wild-type Oregon flies were incubated at $18{ }^{\circ} \mathrm{C}$ and $29^{\circ} \mathrm{C}$ for three days. 24 flies were homogenized following $\mathrm{CO}_{2}$-anesthesy in $500 \mu$ of TRIzol@ Reagent (Invitrogen) according to the manufacturer's instructions. To remove residual genomic DNA, RNA samples (5 $\mu \mathrm{g}$ aliquots) were incubated for 30 min at $37^{\circ} \mathrm{C}$ with 1 unit of DNase (Ambion DNA-free), inactivated at room temperature for two minutes by adding the inactivation reagent. RNeasy 
columns (Qiagen) were applied for further purification. $260 / 280$ and $260 / 230$ ratios in all samples were above 2. Agilent microarray analysis was performed using the commercially available $4 \times 44 \mathrm{k}$ Drosophila platform (Agilent ID: 018,972). $5 \mu \mathrm{g}$ of total RNA was reverse transcribed and used for each condition according to the Agilent Two-color Quick Amp Kit protocol. A technical duplicate was made by dye-swap resulting in eight samples in the following combinations:

\begin{tabular}{llll}
\hline Array 1_1 & \multicolumn{1}{c}{ Array 1_2 } & \multicolumn{1}{c}{ Array 1_3 } & \multicolumn{1}{c}{ Array 1_4 } \\
\hline Ore $20{ }^{\circ} \mathrm{C} / \mathrm{Cy} 5$ & Ore $29{ }^{\circ} \mathrm{C} / \mathrm{Cy} 5$ & Ore $20{ }^{\circ} \mathrm{C} / \mathrm{Cy} 3$ & Ore $29{ }^{\circ} \mathrm{C} / \mathrm{Cy} 3$ \\
DTS-L3 & DTS-L3 & DTS-L3 & DTS $-\angle 3$ \\
$20^{\circ} \mathrm{C} / \mathrm{Cy} 3$ & $29{ }^{\circ} \mathrm{C} / \mathrm{Cy} 3$ & $20^{\circ} \mathrm{C} / \mathrm{Cy} 5$ & $29^{\circ} \mathrm{C} / \mathrm{Cy} 5$ \\
\hline
\end{tabular}

Labeled samples were hybridized in $2 \times$ GEx Hybridization buffer HI-RPM to the array at $65^{\circ} \mathrm{C}, 10$ RPM, for $17 \mathrm{~h}$. The slides were washed sequentially with GE Wash Buffer 1 and 2 and scanned by Agilent G2505B scanner. Microarray data were processed from raw data image files and were quantified with Feature Extraction Software 8.5 (Agilent Technologies). The raw processed signal intensities were normalized to total intensity for comparison between subarrays achieved by Cy5 and Cy3 dyes. Data were filtered by four features: "green is positive and significant", "red is positive and significant", "green is well above background" and "red is well above background". Log2 transformation of normalized values, two sample t-test with equal variances, filtering for $p<0,01$ and averaging of repeated log2 values were calculated. Processed data were subsequently imported and evaluated by the GeneSpring GX and Microsoft Excel. AMIGO gene ontology database was used to find corresponding GO terms; GO annotations were translated by www.sigenae.org.

Validating of the microarray data was achieved using the same templates by QRT-PCR. RevertAid reverse transcriptase (Fermentas) with Random primers (Invitrogen) that were applied for cDNA synthesis and RNaseOut (Invitrogen) was added to avoid RNA degradation. KAPA Sybr Fast Universal qPCR mastermix was used and the reactions were run on an Applied Biosystems 7500 Real-Time PCR System. Expression values in samples of wild-type control flies incubated at $20^{\circ} \mathrm{C}$ served as references. Ct values were corrected with reaction efficiencies and cDNA quantity. Student's t-test was used to calculate statistical significance.

\section{Genotyping of gut bacteria}

Mutant and wild-type flies were incubated for three days at $20^{\circ} \mathrm{C}$ or $29^{\circ} \mathrm{C}$, surfaces were sterilized in $70 \% \mathrm{EtOH}$, guts were dissected from a group of five anesthetized flies and homogenized in $100 \mu$ sterile SB broth. $80 \mu \mathrm{l} 100$-fold dilutions of the suspensions were plated on mannitol-agar (2.5\% n-mannitol,
$0.5 \%$ yeast extract, $0.3 \%$ peptone, $2 \%$ agar, $w / v)$, on SB-agar plates $(3.2 \%$ peptone, $2 \%$ yeast extract, $0.5 \% \mathrm{NaCl}, 2 \%$ agar) and on MRS agar (Scharlau; $\mathrm{g} / \mathrm{l}$ : Peptone proteose 10.00, Meat extract 8.00, Yeast extract $4.00, \mathrm{D}(+)$-Glucose 20.00 , Sodium acetate 5.00 , Triammonium citrate 2.00 , Magnesium sulfate 0.20 , Manganese sulfate 0.05 , Dipotassium phosphate 2.00 , Polysorbate 801.00 , Agar 14.0) incubated at $25^{\circ} \mathrm{C}$. MRS agar is used to grow Lactobacilli; Acetobacter can also grow on this medium but forms smaller colonies. The opposite size distribution can be observed on mannitol plates which is used mainly to grow Acetobacter species. Feeding of bacteria to flies was achieved in both lines kept either at $29^{\circ} \mathrm{C}$ or at $20^{\circ} \mathrm{C}$ by adding $0.1 \mathrm{ml}$ of the formerly isolated Lactobacillus or Acetobacter species at an optical density of 1 at $600 \mathrm{~nm}$ directly to the surface of the food. Clearly distinct bacterial colonies from mannitol-agar plates were pinched and suspended in the PCR-premix for colony PCR. The primers specific for $16 S$ rRNA were 8FE (5'-AGA GTT TGA TCM TGG CTC AG-3') and 1492R (5'-GGM TAC CTT GTT ACG ACT T-3') [13]. ITS1 (TCCGTAGGTGAACCTGCGG) and ITS4 (TCCTCCGCTTATTGATATGC) primers were used for fungal DNA amplification [35]. The products were checked on agarose gel and outsourced for sequencing by Macrogen (Seoul, R. Korea) or to Eurofins MWG Operon (Ebersberg, F.R. Germany).

\section{TUNEL-labeling, ATT::GFP expression, immuno- histochemistry}

Terminal deoxiribonucleotide transferase-mediated dUTP-fluoresceine conjugate nick end labeling (TUNEL) was achieved by in situ cell death detection kit (Roche) as recommended. Embryos of mutant and control flies were incubated at $20^{\circ} \mathrm{C}$ or $29^{\circ} \mathrm{C}$ and L3-stage larvae collected. Nuclei in the dissected guts were counter-stained by $1 \mu \mathrm{g} / \mathrm{ml} \mathrm{4}$, 6-diamidino-2phenylindole (DAPI).

Equimolar mixture of anti-integrin PS I alpha and PSII alpha monoclonal antibodies (mouse, Developmental Studies Hybridoma Bank) were utilized for integrin staining. Mouse monoclonal anti-COL4A1 antibody was raised against the peptide ATGAGSIQDS (29-38) by Creative Ltd., Szeged, Hungary. Primary mouse antibodies were visualized by antimouse Alexa Fluor 488-conjugated secondary antibody (Invitrogen, Life Technology). Laminin gamma 1 polyclonal antibody (rabbit, Abcam) treatment was followed AlexaFluor 568-conjugated anti-rabbit secondary antibody. Experimental procedures described [8] were followed.

Freshly hatched control and mutant adults expressing the Att:GFP transgene were incubated at permissive and restrictive temperatures for three days, guts were dissected. Labellings were visualized by fluorescence microscopy using GFP, FITC or DAPI filters. 


\section{Determination of oxidant concentrations}

Fifty adult flies were homogenized in $450 \mu$ volume of ice-cold phosphate buffered saline solution by means of a plastic homogenizer immersed in an ice water bath, centrifuged at $17,000 \mathrm{~g}$ for $15 \mathrm{~min}$ at $4{ }^{\circ} \mathrm{C}$, and the clear supernatants were used for $\mathrm{H}_{2} \mathrm{O}_{2}$, $\mathrm{ONOO}^{-}$and protein analysis. The quantity of proteins was determined with Folin-Ciocalteu reagent, using bovine serum albumin as standard [36]. Spectrophotometric measurements were carried out with GENESYS 10S UV-Vis spectrophotometers (Thermo Scientific). For the assay of $\mathrm{H}_{2} \mathrm{O}_{2}, 0.05 \mathrm{mg} / \mathrm{mL}$ horseradish peroxidase and $0.1 \mathrm{mg} / \mathrm{mL}$ o-dianisidine in sodium phosphate buffer $(100 \mathrm{mM}, \mathrm{pH} 6.5)$ was used. The $\mathrm{H}_{2} \mathrm{O}_{2}$ concentration was determined spectrophotometrically at $400 \mathrm{~nm}$ and was calculated in $\mathrm{nmol} / \mathrm{mg}$ protein units [37]. $\mathrm{ONOO}^{-}$was assayed by diluting samples in $1 \mathrm{M} \mathrm{NaOH}(60: 1)$ and measuring the increase in absorbance at $302 \mathrm{~nm}$. As a control, samples were added to $100 \mathrm{mM}$ potassium phosphate (pH 7.4) (60:1). The decrease in absorbance was measured at neutral $\mathrm{pH}$ as $\mathrm{ONOO}^{-}$decomposes [38]. $\mathrm{ONOO}^{-}$concentration was expressed in $\mathrm{nmol} / \mathrm{mg}$ protein units.

Supplementary data to this article can be found online at http://dx.doi.org/10.1016/j.matbio.2015.09. 002.

\section{Disclosure}

The authors have no conflicting interests.

\section{Acknowledgments}

This research was supported by the Hungarian Scientific Research Fund OTKA, contract nr. NN 108283 to M.M. and by the European Union and the State of Hungary, co-financed by the European Social Fund in the framework of TÁMOP-4.2.4.A/ 2-11/1-2012-0001 'National Excellence Program' to M.K. The assistance of Prof. A.H. Meijer (Leiden Univ.) during the development of this manuscript is cordially acknowledged.

Received 10 April 2015; Received in revised form 7 September 2015; Accepted 7 September 2015 Available online 10 September 2015

Keywords:

Type IV collagen; Drosophila; Immunity; Gut epithelia

\section{References}

[1] M.A. Morrissey, D.R. Sherwood, An active role for basement membrane assembly and modification in tissue sculpting, J. Cell Sci. 128 (2015) 1661-1668.

[2] A.C. Alport, Hereditary familial congenital haemorrhagic nephritis, Br. Med. J. 1 (1927) 504-506.

[3] K. Tryggvason, J. Zhou, S.L. Hostikka, T.B. Shows, Molecular genetics of Alport syndrome, Kidney Int. 43 (1993) 38-44.

[4] D.B. Gould, F.C. Phalan, G.J. Breedveld, S.E. van Mil, R.S. Smith, J.C. Schimenti, et al., Mutations in Col4a1 cause perinatal cerebral hemorrhage and porencephaly, Science 308 (2005) 1167-1171.

[5] E. Plaisier, O. Gribouval, S. Alamowitch, B. Mougenot, C. Prost, M.C. Verpont, et al., COL4A1 mutations and hereditary angiopathy, nephropathy, aneurysms, and muscle cramps, N. Engl. J. Med. 357 (2007) 2687-2695.

[6] C. Labelle-Dumais, D.J. Dilworth, E.P. Harrington, M. de Leau, D. Lyons, Z. Kabaeva, et al., COL4A1 mutations cause ocular dysgenesis, neuronal localization defects, and myopathy in mice and Walker-Warburg syndrome in humans, PLoS Genet. 7 (2011), e1002062.

[7] D.S. Kuo, C. Labelle-Dumais, D.B. Gould, COL4A1 and COL4A2 mutations and disease: insights into pathogenic mechanisms and potential therapeutic targets, Hum. Mol. Genet. 21 (2012) R97-110.

[8] I. Kelemen-Valkony, M. Kiss, J. Csiha, A. Kiss, U. Bircher, J. Szidonya, et al., Drosophila basement membrane collagen col4a1 mutations cause severe myopathy, Matrix Biol. 31 (2012) 29-37.

[9] I. Kelemen-Valkony, M. Kiss, K. Csiszar, M. Mink, Inherited Myopathies, Nova Publishers, 2012 1-40.

[10] L.V. Hooper, J.I. Gordon, Commensal host-bacterial relationships in the gut, Science 292 (2001) 1115-1118.

[11] P. Tzou, S. Ohresser, D. Ferrandon, M. Capovilla, J.M. Reichhart, B. Lemaitre, et al., Tissue-specific inducible expression of antimicrobial peptide genes in Drosophila surface epithelia, Immunity 13 (2000) 737-748.

[12] R.T. Jones, L.G. Sanchez, N. Fierer, A cross-taxon analysis of insect-associated bacterial diversity, PLoS One 8 (2013), e61218.

[13] J.-H.H. Ryu, S.-H.H. Kim, H.-Y.Y. Lee, J.Y. Bai, Y.-D.D. Nam, J.-W.W. Bae, et al., Innate immune homeostasis by the homeobox gene caudal and commensal-gut mutualism in Drosophila, Science 319 (2008) 777-782.

[14] S.W. Roh, Y.-D.D. Nam, H.-W.W. Chang, K.-H.H. Kim, M.-S.S. Kim, J.-H.H. Ryu, et al., Phylogenetic characterization of two novel commensal bacteria involved with innate immune homeostasis in Drosophila melanogaster, Appl. Environ. Microbiol. 74 (2008) 6171-6177.

[15] E.-M.M. Ha, C.-T.T. Oh, Y.S. Bae, W.-J.J. Lee, A direct role for dual oxidase in Drosophila gut immunity, Science 310 (2005) 847-850.

[16] B. Lemaitre, J. Hoffmann, The host defense of Drosophila melanogaster, Annu. Rev. Immunol. 25 (2007) 697-743.

[17] S. Panayidou, Y. Apidianakis, Regenerative inflammation: lessons from Drosophila intestinal epithelium in health and disease, Pathogens 2 (2013) 209-231.

[18] M. Rera, S. Bahadorani, J. Cho, C.L. Koehler, M. Ulgherait, J.H. Hur, et al., Modulation of longevity and tissue homeostasis by the Drosophila PGC-1 homolog, Cell Metab. 14 (2011) 623-634. 
[19] M. Rera, R.I. Clark, D.W. Walker, Intestinal barrier dysfunction links metabolic and inflammatory markers of aging to death in Drosophila, Proc. Natl. Acad. Sci. U. S. A. 109 (2012) 21528-21533.

[20] F. Bonnay, E. Cohen-Berros, M. Hoffmann, S.Y. Kim, G.L. Boulianne, J.A. Hoffmann, et al., Big bang gene modulates gut immune tolerance in Drosophila, Proc. Natl. Acad. Sci. U. S. A. 110 (2013) 2957-2962.

[21] O. Puig, M.T. Marr, M.L. Ruhf, R. Tjian, Control of cell number by Drosophila FOXO: downstream and feedback regulation of the insulin receptor pathway, Genes Dev. 17 (2003) 2006-2020.

[22] N.A. Broderick, N. Buchon, B. Lemaitre, Microbiota-induced changes in Drosophila melanogaster host gene expression and gut morphology, MBio. 5 (2014) (e01117-14).

[23] N.-H.H. Choi, J.-G.G. Kim, D.-J.J. Yang, Y.-S.S. Kim, M.-A.A. Yoo, Age-related changes in Drosophila midgut are associated with PVF2, a PDGF/VEGF-like growth factor, Aging Cell 7 (2008) 318-334.

[24] L. Sorokin, The impact of the extracellular matrix on inflammation, Nat. Rev. Immunol. 10 (2010) 712-723.

[25] B. Altincicek, A. Vilcinskas, Metamorphosis and collagen-IVfragments stimulate innate immune response in the greater wax moth, Galleria mellonella, Dev. Comp. Immunol. 30 (2006) 1108-1118.

[26] B. Altincicek, A. Berisha, K. Mukherjee, B. Spengler, A. Römpp, A. Vilcinskas, Identification of collagen IV derived danger/alarm signals in insect immunity by nanoLC-FTICR MS, Biol. Chem. 390 (2009) 1303-1311.

[27] R.O. Hynes, Q. Zhao, The evolution of cell adhesion, J. Cell Biol. 150 (2000) F89-F96.

[28] J. Molnar, Z. Ujfaludi, S.F. Fong, J.A. Bollinger, G. Waro, B. Fogelgren, et al., Drosophila lysyl oxidases dmloxl-1 and dmloxl-2 are differentially expressed and the active DmLOXL-1 influences gene expression and development, J. Biol. Chem. 280 (2005) 22977-22985.

[29] J. Molnar, K.S. Fong, Q.P. He, K. Hayashi, Y. Kim, S.F. Fong, et al., Structural and functional diversity of lysyl oxidase and the LOX-like proteins, Biochim. Biophys. Acta 1647 (2003) 220-224.

[30] J.E. Linder, K.A. Owers, D.E. Promislow, The effects of temperature on host-pathogen interactions in D. melanogaster: who benefits? J. Insect Physiol. 54 (2008) 297-308.

[31] N. Buchon, N.A. Broderick, S. Chakrabarti, B. Lemaitre, Invasive and indigenous microbiota impact intestinal stem cell activity through multiple pathways in Drosophila, Genes Dev. 23 (2009) 2333-2344.

[32] S.C. Shin, S.-H.H. Kim, H. You, B. Kim, A.C. Kim, K.-A.A. Lee, et al., Drosophila microbiome modulates host developmental and metabolic homeostasis via insulin signaling, Science 334 (2011) 670-674.

[33] F.L. Connor, C. Di Lorenzo, Chronic intestinal pseudoobstruction: assessment and management, Gastroenterology 130 (2006) S29-S36.

[34] E. Pöschl, U. Schlötzer-Schrehardt, B. Brachvogel, K. Saito, Y. Ninomiya, U. Mayer, Collagen IV is essential for basement membrane stability but dispensable for initiation of its assembly during early development, Development 131 (2004) 1619-1628.

[35] T. White, T. Bruns, S. Lee, J. Taylor, M. Innis, D. Gelfand, et al., Amplification and Direct Sequencing of Fungal Ribosomal RNA Genes for Phylogenetics, Academic Press, San Diego, 1990 315-322.

[36] O.H. LOWRY, N.J. ROSEBROUGH, A.L. FARR, R.J. RANDALL, Protein measurement with the Folin phenol reagent, J. Biol. Chem. 193 (1951) 265-275.

[37] E. Villegas, S. Gilliland, Hydrogen peroxide production by lactobacillus Delbrueckiisubsp. Lactis I at $5^{\circ} \mathrm{C}$, J. Food Sci. 63 (1998) 1070-1074.

[38] R.E. Huie, S. Padmaja, The reaction of no with superoxide, Free Radic. Res. Commun. 18 (1993) 195-199.

[39] P. Tzou, S. Ohresser, D. Ferrandon, M. Capovilla, J.M. Reichhart, B. Lemaitre, J.A. Hoffmann, J.L. Imler, Tissuespecific inducible expression of antimicrobial peptide genes in Drosophila surface epithelia, Immunity 13 (5) (2000) 737-748. 